\title{
Immunohistochemical screening for the diagnosis of succinate dehydrogenase-deficient renal cell carcinoma and fumarate hydratase-deficient renal cell carcinoma
}

\author{
Kiril Trpkov, Farshid Siadat \\ University of Calgary and Alberta Public Labs, Calgary, Alberta, Canada \\ Correspondence to: Kiril Trpkov, MD, FRCPC. Department of Pathology and Laboratory Medicine, University of Calgary, Rockyview General \\ Hospital, 700714 Street, Calgary, AB, Canada. Email: kiril.trpkov@albertapubliclabs.ca. \\ Provenance: This is an invited article commissioned by the Section Editor Dr. Xiao Li (Department of Urology, Jiangsu Cancer Hospital, Jiangsu \\ Institute of Cancer Research, Nanjing Medical University Affiliated Cancer Hospital, Nanjing, China). \\ Comment on: Gupta S, Swanson AA, Chen YB, et al. Incidence of succinate dehydrogenase and fumarate hydratase-deficient renal cell carcinoma \\ based on immunohistochemical screening with SDHA/SDHB and FH/2SC. Hum Pathol 2019;91:114-22.
}

Submitted Sep 03, 2019. Accepted for publication Sep 26, 2019.

doi: 10.21037/atm.2019.09.143

View this article at: http://dx.doi.org/10.21037/atm.2019.09.143

Renal tumors represent heterogeneous and diverse group of neoplasms. The 2013 Vancouver classification of renal tumors and the subsequent 2016 WHO classification represent the foundation of our current knowledge on renal tumors (1). Recent advances have significantly contributed to expanding the morphologic, immunohistochemical, molecular, epidemiologic and clinical features of several novel and emerging renal tumors (2). For example, new evidence has recently emerged on two renal entitiessuccinate dehydrogenase (SDH)-deficient renal cell carcinoma (RCC) and fumarate hydratase-deficient RCC (FH-deficient RCC). The awareness of these novel renal neoplasms is not only essential for practicing pathologists, but also for clinicians and for patient management, because the navigation through this complex and evolving field is a challenging one, even in centers with large volumes of renal tumors. Importantly, the recognition of these novel renal entities rests mainly on their morphologic recognition, with the aid of immunohistochemistry (IHC).

In a recent study performed at the Mayo Clinic, Rochester, MN, USA, Gupta et al. have evaluated their institutional incidence of SDH-deficient RCC and FHdeficient RCC, by applying an IHC based screening with SDHA/SDHB and FH/S-2-succino-cysteine (2SC), employed as IHC-based screening strategies (3). Tissue microarrays were constructed from a cohort of previously diagnosed 1,009 renal cell neoplasms, which were evaluated for SDHA and SDHB. A smaller subset of renal tumors $(\mathrm{n}=730)$, were investigated for $\mathrm{FH}$ deficiency using a combined IHC panel of FH and 2SC. Loss of SDHA and SDHB was found in 3 of 273 tumors that were originally diagnosed as oncocytomas (1.1\%). Nuclear and cytoplasmic 2SC staining with retained $\mathrm{FH}$ expression was found in only one case, while true absence of FH expression was seen in 4 cases: in 2 of 400 papillary RCCs $(0.5 \%)$, and in 2 of 46 unclassified RCC (4.35\%), respectively. No abnormal FH/2SC expression was identified in the 273 cases originally diagnosed as oncocytoma. This study provided additional data to establish the incidence of these rare novel tumors in a series of cases that are specific in terms of subtype, in which one should be particularly vigilant not to miss them. The study also highlights the use of routine screening strategies in the diagnosis of these rare tumors.

In this editorial commentary, we would like to summarize the current data and provide an update on these two renal neoplasms, in the context of the results presented in this study.

\section{SDH-deficient RCC}

SDH-deficient RCC is typically identified in patients with germline mutations in one of the $S D H$ gene subunits: $S D H B$ (great majority), and $S D H A$ or $S D H C$ (4-7). The 

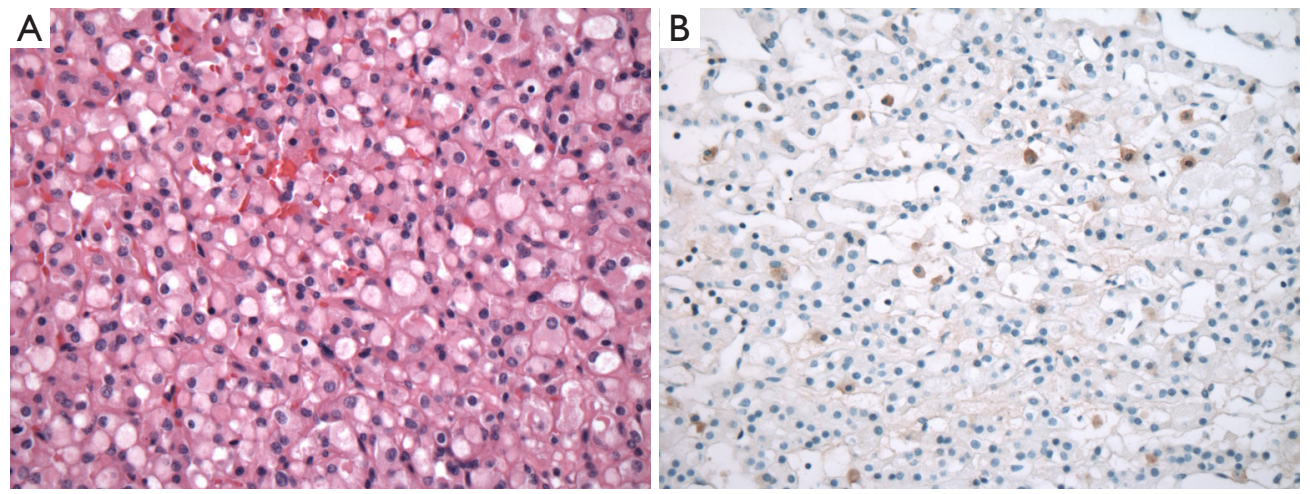

Figure 1 SDH-deficient RCC. (A) Tumor cells in SDH-deficient RCC show diffuse growth and have eosinophilic cytoplasm with wispy, flocculent appearance and low-grade nuclei. Intracytoplasmic vacuoles are also frequently seen and are a helpful diagnostic feature (HE, $\times 200$ ); (B) on IHC, the tumor cells lack staining for SDHB; rare positive mast cells can be identified in the background (IHC, $\times 200$ ). IHC, immunohistochemistry; SDH, succinate dehydrogenase; RCC, renal cell carcinoma.

fundamental finding in establishing a diagnosis of $\mathrm{SDH}$ deficient RCC is the lack of IHC expression for SDHB, owing to bi-allelic inactivation of any of the components of the SDH enzymatic complex, which is located in the mitochondria. SDH complex represents an essential respiratory enzyme that is involved in the normal aerobic respiration, in which it catalyzes the reversible oxidation of succinate into fumarate, and it also participates in the electron transport chain $(6,7)$.

It has now been accepted that an absence of the SDHB protein expression detected by IHC can be utilized to screen for syndromic disease, which is typically associated with a germline mutation of any of the SDH subunits A to D (4-7). Tumors that exhibit an absence of SDHB reactivity are named "SDH-deficient", and besides SDH-deficient RCC, also include SDH-deficientpheochromocytoma, paraganglioma, gastrointestinal stromal tumor (GIST), and pituitary adenoma $(6,7)$. Patients with SDH-deficient RCC may also demonstrate a hereditary paraganglioma-pheochromocytoma syndrome, and may exhibit a family history of RCC, paraganglioma or GIST. Such findings in the clinical or the family history should initiate patient genetic testing for germline $S D H$ mutations. A diagnosis of SDH-deficient RCC therefore mandates a follow-up and surveillance of the patient, as well as genetic counselling and evaluation of the other family members. In our view, IHC in this setting represents a phenotypic test rather than a genotypic one, which generally does not require a consent or formal genetic counseling, before IHC is done. A parallel can be drawn to the analogous IHC evaluation for DNA mismatch repair proteins, which is used in patients with colorectal cancer that are tested for Lynch syndrome.

The great majority of the documented cases with typical features of SDH-deficient renal carcinomas have demonstrated germline mutations for $S D H B$ and $S D H C$, which is exemplified by a loss of SDHB expression on IHC $(4,5)$. To date, only rare renal carcinoma cases have been documented with $S D H A$ mutation, which typically show an IHC loss of SDHA, in addition to the loss of SDHB (8).

SDH-deficient RCC are uncommon tumors that were documented in $0.05-0.2 \%$ of all renal carcinoma in individual institutional cohorts $(4,5,9)$. In the largest reported series, the mean age of the patients with SDHdeficient RCC was 38 years (range, 14-76 years), with a male to female ratio of 1.8 to $1(4,9)$. SDH-deficient RCC are typically solitary tumors, but can also occur as bilateral and multifocal tumors in up to a third of patients (4). Most tumors are organ confined, with an average size of $5.1 \mathrm{~cm}$ (range, 0.7-9 cm) $(4,9)$. Because SDH-deficient RCC are rare, it is not practical to do reflex IHC screening on all renal neoplasms, but it is important to be familiar with the stereotypical features of SDH-deficient RCCs, so that appropriate testing can be done when necessary. SDH-deficient RCCs demonstrate a typical morphology, illustrated in Figure 1. SDH-deficient RCC are well circumscribed, non-encapsulated tumors with diffuse sheetlike or compact nested growth, and focal cystic change is common. The neoplastic cells show eosinophilic, flocculent or wispy cytoplasm, lacking the fine cytoplasmic granularity, typically seen in the tumor that represents their main differential diagnosis-renal oncocytoma; they also lack 
the prominent cell borders, as seen in chromophobe RCC. Frequent intracytoplasmic inclusions or vacuoles are also quite frequent, although in some cases they can be difficult to find. Other characteristic IHC features include lack of reactivity for $\mathrm{CD} 117$, and frequent absence or weak and focal cytokeratin reactivity.

The overall risk for metastatic disease is about $10 \%$ and late metastases may occur, even many years after the initial resection of the SDH-deficient RCC $(4,5)$. High-grade transformation, present in about a third of the cases, along with coagulative necrosis and sarcomatoid transformation are all considered adverse findings that result in metastatic disease in about $70 \%$ of cases with such features (4). These cases although unrecognizable as typical SDH-deficient RCC on morphology, can also be identified by IHC for SDHB.

\section{FH-deficient RCC}

FH-deficient RCC represents an RCC that typically presents with advanced disease and is characterized by very aggressive behavior $(10,11)$. FH-deficient RCC demonstrates a germline mutation in the $\mathrm{FH}$ gene, located at chromosome 1q42.3-q43 (12-14). The FH enzyme is also involved in the Krebs cycle and catalyzes fumarate to form malate; when mutated, it leads to hereditary leiomyomatosis and RCC syndrome (HLRCC) (12-14). Fumarate accumulation acts as a metabolic tumor suppressor and initiates a cascade of biochemical reactions that lead to development of renal carcinomas that are designated as "FH-deficient RCC", as well as "HLRCC-associated RCC", when the patient syndromic characteristics are known. "FH-deficient RCC" is a terminology that more specifically refers to an RCC that shows: (I) compatible morphology, (II) IHC-negative reactivity for $\mathrm{FH}$, and/or IHC- reactivity for 2SC, (III) uncertain clinical and family history regarding possible cutaneous and uterine leiomyomas and RCC, and (IV) unknown genetic status at the time of the initial presentation $(10,11)$.

FH-deficient RCC is very similar to the kidney cancers found in the HLRCC syndrome, which represents an autosomal dominant hereditary disease, characterized by uterine and skin leiomyomas and RCCs (12-14). FHdeficient RCC and HLRCC-associated RCC are found more frequently in younger patients, and demonstrate aggressive course and adverse features at the time of presentation. They typically exhibit multiple morphologic patterns, most often papillary pattern, and often focal, viral-like macronucleoli (Figure $2 A, B)(10,11,15-17)$. IHC screening should particularly be done in renal tumors that are considered "unclassified, high grade", "unclassified with dominant papillary pattern" "tubulocystic carcinoma with dedifferentiated (or solid) foci" "papillary type 2", or "collecting duct carcinoma" $(10,11)$.

$\mathrm{FH}$ gene mutation results in either complete loss or reduction of the $\mathrm{FH}$ enzymatic activity that results in accumulation of intracellular fumarate, with an increased protein succination and accumulation of $2 \mathrm{SC}$. On IHC, 2SC demonstrates strong immunoreactivity that is highly sensitive in FH-deficient tumors $(10,11,15-18)$. The loss of $\mathrm{FH}$ enzymatic activity produces an absence of $\mathrm{FH}$ expression on IHC, which is highly specific in identifying these tumors (10,11,15-18). A combined IHC testing for $2 \mathrm{SC}$ and $\mathrm{FH}$, as done in the study by Gupta et al., can therefore significantly facilitate the detection of $\mathrm{FH}$ deficient RCC (Figure 2C,D) (3). Currently, FH-deficient RCC is generally screened for by utilizing the negative IHC result for $\mathrm{FH}$. IHC evaluation for 2SC, which produces a positive result (either nuclear or nuclear/cytoplasmic expression), is apparently a more sensitive test, but so far, it was used in a more restrictive fashion, because 2SC antibody became commercially available very recently $(10,11,15-18)$.

The identification of FH-deficient $\mathrm{RCC}$ is very important, not only because they are very aggressive tumors, but also due to their hereditary association. When such tumors are diagnosed, a genetic counseling and $\mathrm{FH}$ mutational analysis in the patients and their families should be performed. If $F H$ mutations are detected in any family members, an ongoing surveillance would optimally include annual abdominal magnetic resonance imaging (19). Any newly identified renal tumors in these patients should be treated promptly, with a wide-margin surgical resection and with possible retroperitoneal resection of the lymph nodes (10).

In our recent study, patients with $\mathrm{FH}$-deficient RCC had a median age of 44 years, and were more commonly males (male: female $=1.9: 1$ ) (10). The tumors were large size, typically solitary and unilateral (mean size $8.2 \mathrm{~cm}$; range, 0.9 to $18 \mathrm{~cm}$ ), demonstrating a high-stage disease at presentation $(57 \%$ stage $\geq \mathrm{pT} 3,52 \%$ with positive nodes, and $19 \%$ with distant metastases) (10). The prognosis was dismal and after a mean follow-up of only 27 months, 39\% of the patients were dead of disease, and $26 \%$ demonstrated 

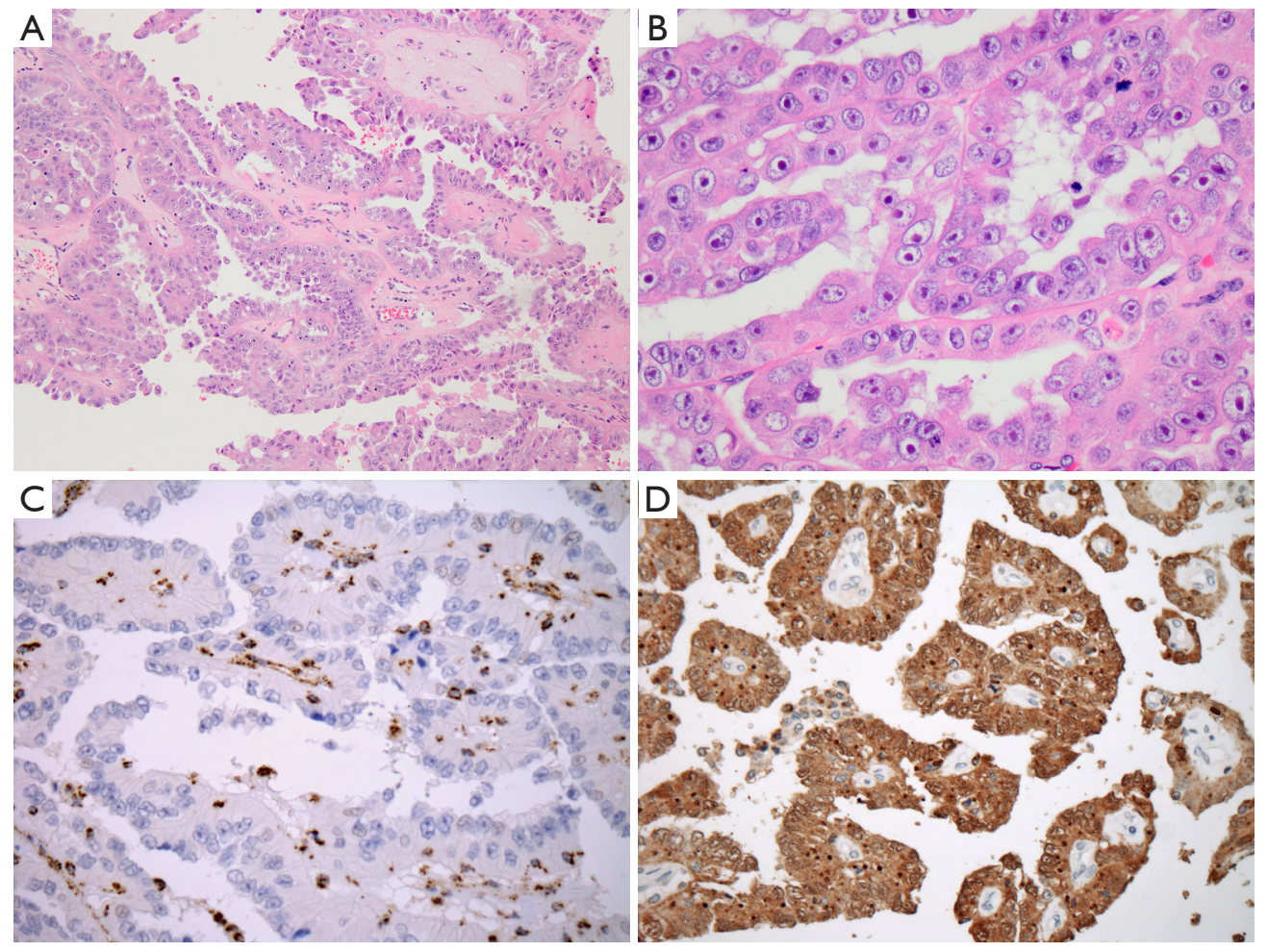

Figure 2 FH-deficient RCC (). (A) FH-deficient RCCs typically demonstrate multiple architectural patterns, but most often a papillary pattern (HE, $\times 100$ ); (B) large eosinophilic nucleoli can be found at least focally, but they should not be considered specific (HE, $\times 400)$; (C) negative staining for $\mathrm{FH}$ (only the stromal cells in the background are positive) supports the diagnosis (IHC, $\times 400$ ); (D) positive staining for $2 \mathrm{SC}$ is also helpful in establishing the diagnosis (IHC, $\times 400)$. IHC, immunohistochemistry; FH, fumarate hydratase; RCC, renal cell carcinoma.

disease progression (10).

Microscopically, the FH-deficient RCC demonstrated presence of 2 or more growth patterns in $93 \%$ of cases (10); papillary morphology was the most common pattern and often dominant, while other common patterns included: solid, tubulocystic, cribriform, and cystic. Several recent studies also confirmed that the constellation of multiple growth patterns was the key morphology that characterized the FH-deficient RCC (11,16-18).

In summary, the accurate diagnosis of SDH-deficient and FH-deficient RCC is of utmost clinical significance, despite their rarity. Therefore, we strongly recommend that IHC-based screening strategies, using SDHA/SDHB and $\mathrm{FH} / 2 \mathrm{SC}$, such as demonstrated by Gupta et al. should be used as standard screening in tertiary or quaternary centers that have large volumes of renal tumors (3). The awareness of these RCCs and their inclusion in the differential diagnosis is particularly important in a subtypespecific setting, which would allow their accurate diagnosis for an appropriate clinical management and patient prognostication.

\section{Acknowledgments}

None.

\section{Footnote}

Conflicts of Interest: The authors have no conflicts of interest to declare.

Ethical Statement: The authors are accountable for all aspects of the work in ensuring that questions related to the accuracy or integrity of any part of the work are appropriately investigated and resolved.

\section{References}

1. Moch H, Humphrey PA, Ulbright TM, et al. WHO classification of tumors of the urinary system and male 
genital organs. 4th Edition ed. Lyon, France: International Agency for Research on Cancer, 2016.

2. Trpkov K, Hes O. New and Emerging Renal Entities: A Perspective Post WHO 2016 Classification. Histopathology 2019;74:31-59.

3. Gupta S, Swanson AA, Chen YB, et al. Incidence of succinate dehydrogenase and fumarate hydratase-deficient renal cell carcinoma based on immunohistochemical screening with SDHA/SDHB and FH/2SC. Hum Pathol 2019;91:114-22.

4. Gill AJ, Hes O, Papathomas T, et al. Succinate dehydrogenase (SDH)-deficient renal carcinoma: A morphologically distinct entity: A clinicopathologic series of 36 tumors from 27 patients. Am J Surg Pathol 2014;38:1588-602.

5. Gill A, Amin M, Smith S, et al. Succinate dehydrogenasedeficient renal carcinoma. In: Moch H, Humphrey $\mathrm{P}$, Ulbright T, et al. (eds). WHO classification of tumors of the urinary system and male genital organs. France: Lyon: International Agency for Research on Cancer, 2016:35.

6. Gill AJ. Succinate dehydrogenase (SDH) and mitochondrial driven neoplasia. Pathology 2012;44:285-92.

7. Gill AJ. Succinate dehydrogenase (SDH)-deficient neoplasia. Histopathology 2018;72:106-16.

8. Yakirevich E, Ali SM, Mega A, et al. A novel SDHAdeficient renal cell carcinoma revealed by comprehensive genomic profiling. Am J Surg Pathol 2015;39:858-63.

9. Williamson SR, Eble JN, Amin MB, et al. Succinate dehydrogenase-deficient renal cell carcinoma: Detailed characterization of 11 tumors defining a unique subtype of renal cell carcinoma. Mod Pathol 2015;28:80-94.

10. Trpkov K, Hes O, Agaimy A, et al. Fumarate hydratasedeficient renal cell carcinoma is strongly correlated with fumarate hydratase mutation and hereditary leiomyomatosis and renal cell carcinoma syndrome. Am J Surg Pathol 2016;40:865-75.

11. Smith SC, Trpkov K, Chen YB, et al. Tubulocystic carcinoma of the kidney with poorly differentiated foci:

Cite this article as: Trpkov K, Siadat F. Immunohistochemical screening for the diagnosis of succinate dehydrogenasedeficient renal cell carcinoma and fumarate hydratase-deficient renal cell carcinoma. Ann Transl Med 2019;7(Suppl 8):S324. doi: 10.21037/atm.2019.09.143
A frequent morphologic pattern of fumarate hydratasedeficient renal cell carcinoma. Am J Surg Pathol 2016;40:1457-72.

12. Kiuru M, Launonen V. Hereditary leiomyomatosis and renal cell cancer (HLRCC). Curr Mol Med 2004;4:869-75.

13. Launonen V, Vierimaa O, Kiuru M, et al. Inherited susceptibility to uterine leiomyomas and renal cell cancer. Proc Natl Acad Sci U S A 2001;98:3387-92.

14. Tomlinson IP, Alam NA, Rowan AJ, et al. Germline mutations in $\mathrm{FH}$ predispose to dominantly inherited uterine fibroids, skin leiomyomata and papillary renal cell cancer. Nat Genet 2002;30:406-10.

15. Merino MJ, Torres-Cabala C, Pinto P, et al. The morphologic spectrum of kidney tumors in hereditary leiomyomatosis and renal cell carcinoma (HLRCC) syndrome. Am J Surg Pathol 2007;31:1578-85.

16. Chen YB, Brannon AR, Toubaji A, et al. Hereditary leiomyomatosis and renal cell carcinoma syndromeassociated renal cancer: Recognition of the syndrome by pathologic features and the utility of detecting aberrant succination by immunohistochemistry. Am J Surg Pathol 2014;38:627-37.

17. Muller M, Ferlicot S, Guillaud-Bataille M, et al. Reassessing the clinical spectrum associated with hereditary leiomyomatosis and renal cell carcinoma syndrome in French FH mutation carriers. Clin Genet 2017;92:606-15.

18. Muller M, Guillaud-Bataille M, Salleron J, et al. Pattern multiplicity and fumarate hydratase $(\mathrm{FH}) / \mathrm{s}$-(2-succino)cysteine (2SC) staining but not eosinophilic nucleoli with perinucleolar halos differentiate hereditary leiomyomatosis and renal cell carcinoma-associated renal cell carcinomas from kidney tumors without $\mathrm{FH}$ gene alteration. Mod Pathol 2018;31:974-83.

19. Menko FH, Maher ER, Schmidt LS, et al. Hereditary leiomyomatosis and renal cell cancer (HLRCC): Renal cancer risk, surveillance and treatment. Fam Cancer 2014;13:637-44. 